\title{
Polyhedration of multi-component systems of nickel with transition metals (V-VIIB subgroups) using the graph method
}

\author{
E.Yu. KERIMOV ${ }^{1}$, Yu.V. BALYKOVA ${ }^{2}$, E.M. SLYUSARENKO ${ }^{2 *}$ \\ ${ }^{1}$ Belinsky Penza State University, Department of Chemistry, Lermontov St.,37, 440026 Penza, Russian Federation \\ ${ }^{2}$ Department of Chemistry, Lomonosov Moscow State University, Leninskie Gory, 119992 Moscow, \\ Russian Federation \\ * Corresponding author. E-mail: slusarenko@laincom.chem.msu.ru
}

Received September 16, 2007; accepted December 29, 2008; available on-line March 19, 2009

The graph method can be used for the determination of phase equilibria in multi-component systems. The determination of phase equilibria in an $n$-component phase diagram using the graph method can be realized on the basis of data on the phase equilibria in (n-1)-component systems. The phase diagrams of the fivecomponent system Ni-Re-V-Cr-Mo at $1425 \mathrm{~K}$, six-component system Ni-Mn-V-Cr-Mo-W at 1225-1250 K and seven-component system Ni-V-Nb-Ta-Cr-Mo-W at $1375 \mathrm{~K}$ have been investigated. All four-component equilibria in these systems have been determined by experimental investigation. Isothermal cross-sections have been constructed for all systems.

\section{Polyhedration / Phase diagram / Transition metals / Graph method}

Phase diagrams are a universal source of information on the interaction of the elements in multi-component systems and serve as the basis for the creation of new materials. However, multi-component diagrams have been insufficiently researched, because the experimental work significantly increases in investigations with many components.

Constructing a phase diagram can be subdivided into two stages:

1) All phases forming in the system and the scheme of phase equilibria are determined. This is the polyhedration of the system.

2) The phase boundaries and directions of tie lines are defined. The obtained information is presented in analytical form.

The performance of the second stage is hard work and often not feasible. The first stage is qualitative and can be solved. The data obtained at this stage are useful for many scientists since they make it possible to choose a fragment of a multi-component system that is perspective for the creation of new materials with special properties.

The graph method [1-3] can be used for the polyhedration of systems (determination of all phases and the scheme of phase equilibria). Any $N$-phase equilibrium may be presented as a complete graph with $N$ vertices (Fig. 1). Each phase is marked as a point (independent of the components), and two vertices are connected to form an edge in the graph (line between points), if an equilibrium exists between these two phases.
The graph containing all equilibria in the system at a chosen temperature represents a formalised isothermal cross-section of the phase diagram and is named total graph.

Polyhedration of an $n$-component phase diagram using the graph method can be realised on the basis of data on the phase equilibria in the (n-1)-component systems by performing three consecutive steps:

1) Presenting isothermal cross-sections of the (n-1)component systems as graphs. Graphs of the simplest isothermal cross-sections of ternary systems are given in Fig. 2. Combination of these graphs gives a formalised isothermal cross-section.

2) Building a graph of the $n$-component system (total graph) by combining the graphs of the (n-1)component systems. The number of vertices (points in a plane) of the obtained graph is equal to the number of phases in the $n$-component system. The edges of the graph are obtained by copying all edges from the source graphs of the $(n-1)$-component systems to the graph of the $n$-component system.

3 ) Decomposing the total graph of the $n$-component system into graphs of $N$ - and $(N-1)$-phase equilibria. However, it is possible to obtain several variants from such a division since, upon decomposition of the graph it is necessary to note that the phase equilibria in the (n-1)-component systems do not give complete information about all phase equilibria in the $n$ component system. An ( $N$-1)-phase equilibrium from the $(n-1)$-component system in the $n$-component system can: 

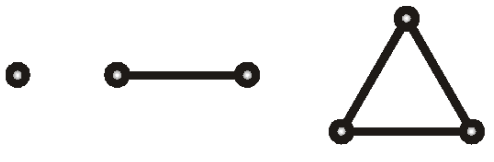

1

3

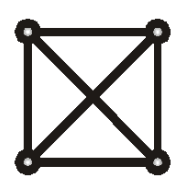

4

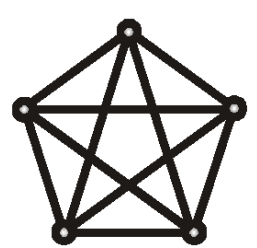

5

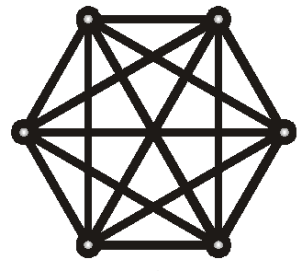

6

Fig. 1 Presentation of multiphase equilibria as graphs.

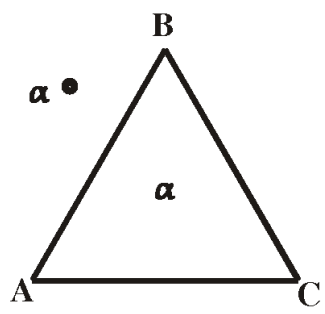

a)

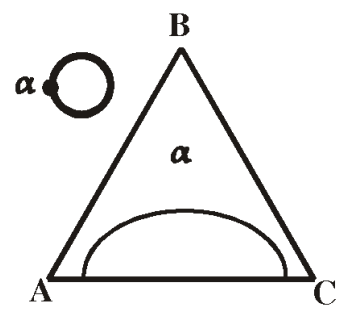

b)

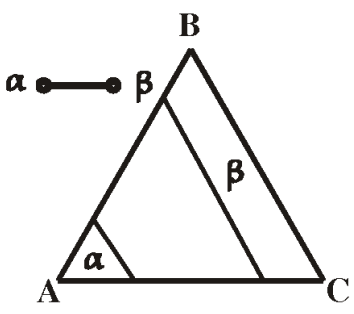

c)

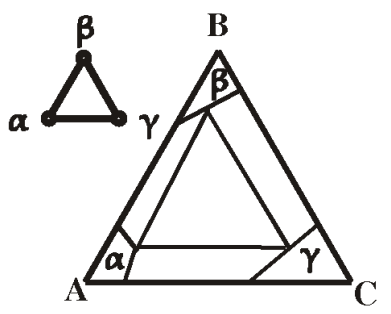

d)

Fig. 2 Presentation of the simplest isothermal cross-sections of ternary systems as graphs: a) a single phase, b) degeneration of equilibria, c) recombination of equilibria, d) formation of an $N$-phase quilibrium.

Table 1 Phase equilibria in the quaternary systems of the quinary Ni-V-Cr-Mo-Re system.

\begin{tabular}{c|c|c|c}
\hline No. & System & Phases & Four-phase equilibria \\
\hline 1 & Ni-V-Cr-Mo & $\beta, \gamma, \sigma, \delta, \rho$ & $\beta+\gamma+\sigma+\rho ; \beta+\gamma+\delta+\rho$ \\
2 & Ni-V-Cr-Re & $\beta, \gamma, \sigma, \operatorname{Re}$ & - \\
3 & Ni-V-Mo-Re & $\beta, \gamma, \sigma, \delta, \chi, \rho, \operatorname{Re}$ & $\beta+\gamma+\sigma+\rho ; \beta+\gamma+\delta+\rho ; \gamma+\sigma+\rho+\operatorname{Re}$ \\
4 & Ni-Cr-Mo-Re & $\beta, \gamma, \sigma, \delta, \chi, \rho, \operatorname{Re}$ & $\gamma+\sigma+\rho+\operatorname{Re}$ \\
5 & V-Cr-Mo-Re & $\beta, \sigma, \chi, \operatorname{Re}$ & - \\
\hline
\end{tabular}

a) degenerate, moving over to an (N-2)-phase equilibrium (Fig. 2b);

b) recombine with an $(N-1)$-phase equilibrium forming an (N-1)-phase field (Fig. 2c);

c) participate in the formation of an $N$-phase equilibrium (Fig. 2d).

Taking into account the aforesaid, the degenerated and recombined (N-1)-phase equilibria should be separated from the total graph. The remaining graph will represent a collection of $N$-phase equilibria. If two or more $N$-phase equilibria form a general block then the remaining graph is indecomposable. This is observed when several $N$-phase equilibria have similar $(N-1)$-phase equilibria in a polyhedron. In this case it is necessary to carry out an experimental investigation.

The different steps of the decomposition of the total graph are, as an example, shown for the Ni-VTa-Cr system (Fig. 3). There are two recombined three-phase equilibria $\lambda_{1}+\lambda_{2}+\beta_{1}$ and $\lambda_{1}+\lambda_{2}+\beta_{2}$ in this system (Fig. 3b,c). Two four-phase equilibria $\mu+\beta+\lambda_{1}+\mathrm{NiTa}_{2}$ and $\alpha+\mu+\lambda_{1}+\mathrm{Ni}_{2} \mathrm{Ta}$ are distinguished as a result of the analysis (Fig. $3 \mathrm{~d}, \mathrm{e}$ ), and a remaining graph consisting of five phases $\left(\sigma, \beta, \lambda_{1}, \alpha, \gamma\right)$ is obtained (Fig. 3f). The investigation of four alloys, melted in the field between these phases, revealed the existence two four-phase equilibria: $\sigma+\gamma+\alpha+\beta$ and $\beta+\sigma+\alpha+\lambda_{1}$ (Fig. 3g,h).

\section{Polyhedration of the system Ni-V-Cr-Mo-Re}

The system Ni-V-Cr-Mo-Re was investigated. It includes ten ternary systems: $\mathrm{V}-\mathrm{Cr}-\mathrm{Mo}, \mathrm{Ni}-\mathrm{Cr}-\mathrm{Re}, \mathrm{Ni}-$ Mo-Re, Ni-V-Re, Ni-Cr-Mo, Ni-V-Cr, Ni-V-Mo, V$\mathrm{Cr}-\mathrm{Re}, \mathrm{V}-\mathrm{Mo}-\mathrm{Re}$, and Cr-Mo-Re. The phase equilibria were determined for the systems Ni-V-Cr-Mo, Ni-V$\mathrm{Cr}-\mathrm{Re}$, Ni-V-Mo-Re, Ni-Cr-Mo-Re, V-Cr-Mo-Re and $\mathrm{Ni}-\mathrm{V}-\mathrm{Cr}-\mathrm{Mo}-\mathrm{Re}$ by using the graph method. The obtained data were confirmed by an experimental investigation.

Three four-phase equilibria occur in the fivecomponent Ni-V-Cr-Mo-Re system, as shown in Table 1. For the formation of a five-phase equilibrium in a quinary system it is necessary to have five different types of four-phase equlibrium. Therefore five-phase equilibria are absent in the five-component Ni-V-Cr-Mo-Re system. 


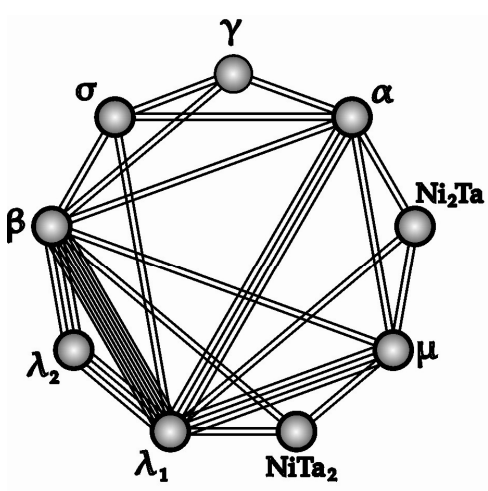

a)

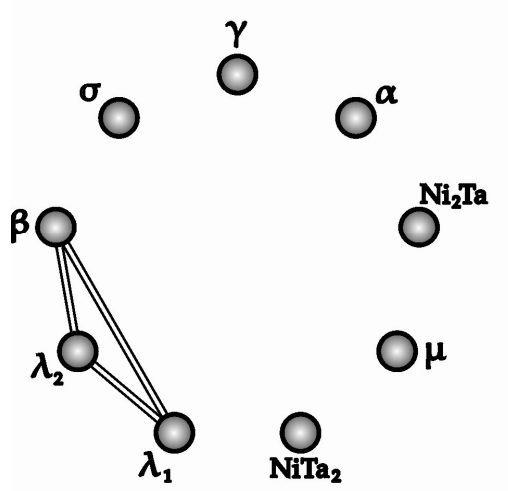

c)

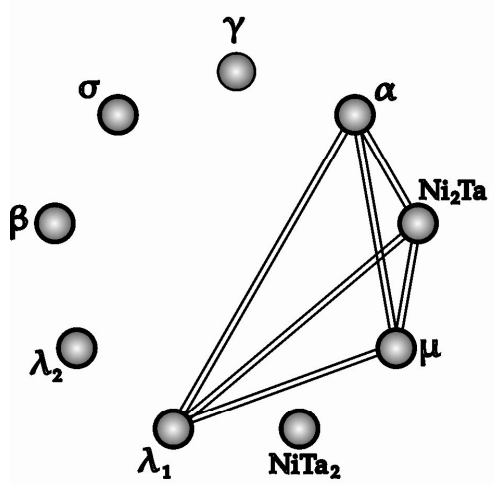

e)

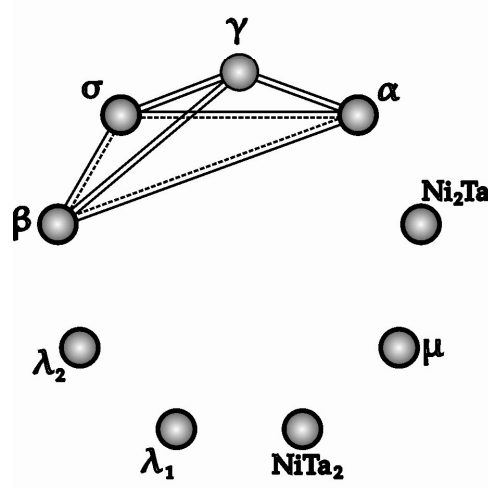

g)

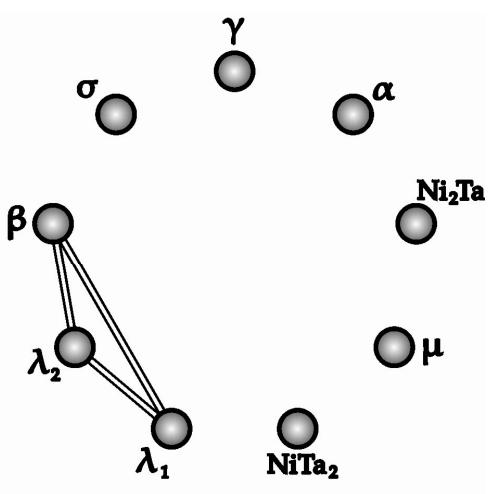

b)

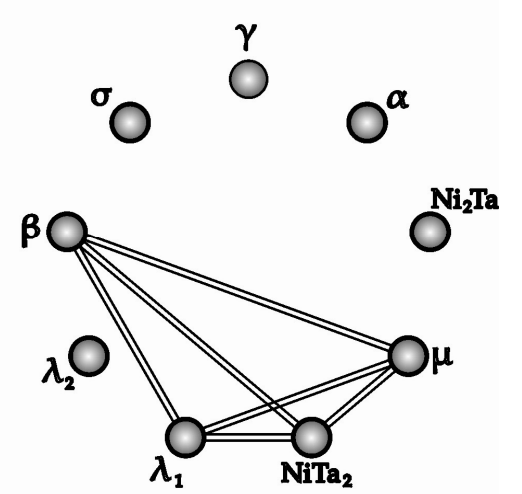

d)

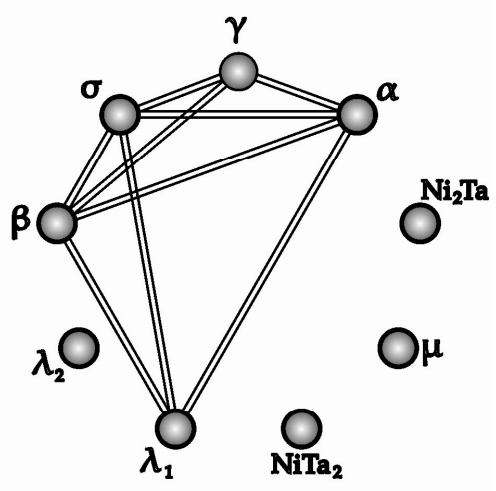

f)

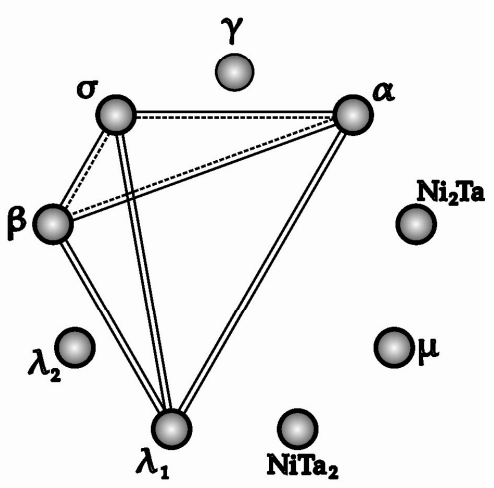

h)

Fig. 3 Polyhedration of the Ni-V-Ta-Cr system: a) total graph of three-phase equilibria, b,c) recombined equilibria, d,e) remaining graphs of zero rank, f) remaining graph of first rank, g,h) its decomposition into four-phase equilibria. 
E.Yu. Kerimov et al., Polyhedration of multi-component systems of nickel with transition metals ...

Table 2 Phase equilibria in the quaternary systems of the Ni-Mn-V-Cr-Mo-W system.

\begin{tabular}{|c|c|c|c|}
\hline No. & System & Phases & Four-phase equilibria \\
\hline 1 & Mn-V-Cr-Mo & $(\beta-\mathrm{Mn}), \beta, \sigma$ & - \\
\hline 2 & $\mathrm{Mn}-\mathrm{V}-\mathrm{Cr}-\mathrm{W}$ & $(\beta-\mathrm{Mn}), \beta, \sigma$ & - \\
\hline 3 & Mn-V-Mo-W & $(\beta-\mathrm{Mn}), \beta, \sigma$ & - \\
\hline 4 & Mn-Cr-Mo-W & $(\beta-\mathrm{Mn}), \beta, \sigma$ & - \\
\hline 5 & V-Cr-Mo-W & $\beta$ & - \\
\hline 6 & $\mathrm{Ni}-\mathrm{Mn}-\mathrm{V}-\mathrm{Cr}$ & $(\beta-\mathrm{Mn}), \beta, \gamma, \sigma$ & - \\
\hline 7 & Ni-Mn-V-Mo & $(\beta-\mathrm{Mn}), \beta, \gamma, \delta, \sigma$ & - \\
\hline 8 & Ni-Mn-V-W & $(\beta-\mathrm{Mn}), \beta, \gamma, \sigma, \mathrm{WNi}_{4}$ & $(\beta-\mathrm{Mn})+\beta+\gamma+\sigma$ \\
\hline 9 & Ni-Mn-Cr-Mo & $(\beta-\mathrm{Mn}), \beta, \gamma, \delta, \sigma, \mathrm{P}$ & $\beta+\gamma+\sigma+\mathrm{P} ; \beta+\gamma+\delta+\mathrm{P}$ \\
\hline 10 & $\mathrm{Ni}-\mathrm{Mn}-\mathrm{Cr}-\mathrm{W}$ & $(\beta-\mathrm{Mn}), \beta, \gamma, \sigma, \mathrm{WNi}_{4}$ & $(\beta-\mathrm{Mn})+\beta+\gamma+\sigma$ \\
\hline 11 & Ni-Mn-Mo-W & $(\beta-\mathrm{Mn}), \beta, \gamma, \delta, \sigma, \mathrm{WNi}_{4}$ & $(\beta-\mathrm{Mn})+\beta+\gamma+\sigma$ \\
\hline 12 & Ni-V-Cr-Mo & $\beta, \gamma, \delta, \sigma, \mathrm{P}$ & $\beta+\gamma+\sigma+\mathrm{P} ; \beta+\gamma+\delta+\mathrm{P}$ \\
\hline 13 & $\mathrm{Ni}-\mathrm{V}-\mathrm{Cr}-\mathrm{W}$ & $\beta, \gamma, \sigma, \mathrm{WNi}_{4}$ & - \\
\hline 14 & Ni-V-Mo-W & $\beta, \gamma, \sigma, \delta, \mathrm{WNi}_{4}$ & - \\
\hline 15 & Ni-Cr-Mo-W & $\beta, \gamma, \sigma, \mathrm{P}, \delta, \mathrm{WNi}_{4}$ & $\beta+\gamma+\sigma+\mathrm{P} ; \beta+\gamma+\delta+\mathrm{P}$ \\
\hline
\end{tabular}

Table 3 Phase equilibria in quaternary systems based on refractory metals.

\begin{tabular}{c|c|c}
\hline Systems & Phases & Phase equilibria \\
\hline V-Nb-Mo-W, Nb-Ta-Mo-W & $\beta$ & - \\
V-Nb-Ta-Cr, V-Nb-Ta-Mo, V-Nb-Ta-W, & $\lambda_{2}, \beta$ & $\lambda_{2}+\beta$ \\
V-Ta-Cr-Mo, V-Ta-Mo-W & $\beta$ & $\begin{array}{c}\text { Region of stratification of the } \beta \text { phase } \\
\text { V-Cr-Mo-W }\end{array}$ \\
$\begin{array}{c}\text { Degeneration of the three-phase equilibrium } \\
\lambda_{2}+\beta_{1}+\beta_{2} \text { to the two-phase equilibrium } \lambda_{2}+\beta \\
\text { Recombination of the three-phase equilibrium } \\
\lambda_{2}+\beta_{1}+\beta_{2}\end{array}$ \\
Nb-Ta-Cr-Mo, Nb-Cr-Mo-W
\end{tabular}

\section{Polyhedration of the system Ni-Mn-V-Cr-Mo-W}

Polyhedration of the six-component system $\mathrm{Ni}-\mathrm{Mn}-\mathrm{V}-\mathrm{Cr}-\mathrm{Mo}-\mathrm{W}$ was realized at $1250 \mathrm{~K}$. Only in six of the quaternary systems four-phase equilibria were established (Table 2). The equilibrium $(\beta-\mathrm{Mn})+\beta+\gamma+\sigma$ was determined in the $\mathrm{Ni}-\mathrm{Mn}-\mathrm{V}-\mathrm{W}$, $\mathrm{Ni}-\mathrm{Mn}-\mathrm{Cr}-\mathrm{W}$ and Ni-Mn-Mo-W systems. The existence of a remaining graph consisting of five phases: $\beta, \gamma, \delta, \sigma, \mathrm{P}$, was discovered in the $\mathrm{Ni}-\mathrm{Mn}-\mathrm{Cr}-\mathrm{Mo}$, Ni-V-Cr-Mo and Ni-Cr-Mo-W systems. This graph was solved earlier by an experimental investigation of the quinary system $\mathrm{Ni}$ $\mathrm{V}-\mathrm{Cr}-\mathrm{Mo}-\mathrm{Re}$. It consists of two four-phase equilibria: $\beta+\gamma+\delta+P$ and $\beta+\gamma+\sigma+P$.

Only three different types of four-component equilibrium exist in the Ni-V-Cr-Mo-W-Mn system. For the formation of a five-phase equlibrium it is necessary to have five different types of four-phase equilibrium. Consequently, in this system at $1250 \mathrm{~K}$ five-phase equilibria are absent.

\section{Polyhedration of the system Ni-V-Nb-Ta-Cr-Mo-W}

For the seven-component system Ni-V-Nb-Ta-Cr-Mo$\mathrm{W}$ the phase equilibria in 35 quaternary systems, 21 quinary systems and 7 six-component systems have been analyzed using the graph method.

The ternary systems (and supposedly also the quaternary systems) without $\mathrm{Ni}$ contain only two phases: a bcc solid solution and a Laves $\lambda_{2}$-phase, which exists in the $\mathrm{V}-\mathrm{Ta}, \mathrm{Cr}-\mathrm{Nb}$ and $\mathrm{Cr}-\mathrm{Ta}$ systems. It follows that in the multi-component systems of the refractory metals only two-phase equilibria can exist.

The Nb-Cr-Mo, Nb-Cr-W, Ta-Cr-Mo and Ta-Cr-W systems are exceptions, because they contain the three-phase equilibrium $\lambda_{2}+\beta_{1}+\beta_{2}$. In spite of the existence of only two phases in these systems, threephase equilibria are present because of the stratification of the $\beta$-solid solution.

Upon addition of a fourth refractory metal these regions form a three-phase field in the quaternary system (in the $\mathrm{Nb}-\mathrm{Ta}-\mathrm{Cr}-\mathrm{W}$ system), or degenerate to a region of two-phase equilibrium (in the remaining systems). A review of the quaternary systems of the metals of groups VB and VIB is given in Table 3.

In the systems on the basis of nickel and refractory metals there are many intermetallic compounds. For the polyhedration of the isothermal cross-sections of the quaternary phase diagrams the graph method was used. Only in twelve diagrams remaining graphs were obtained. Experimental decomposition of the 
E.Yu. Kerimov et al., Polyhedration of multi-component systems of nickel with transition metals ...

Table 4 Four-phase equilibria in quaternary systems on the basis of nickel and refractory metals.

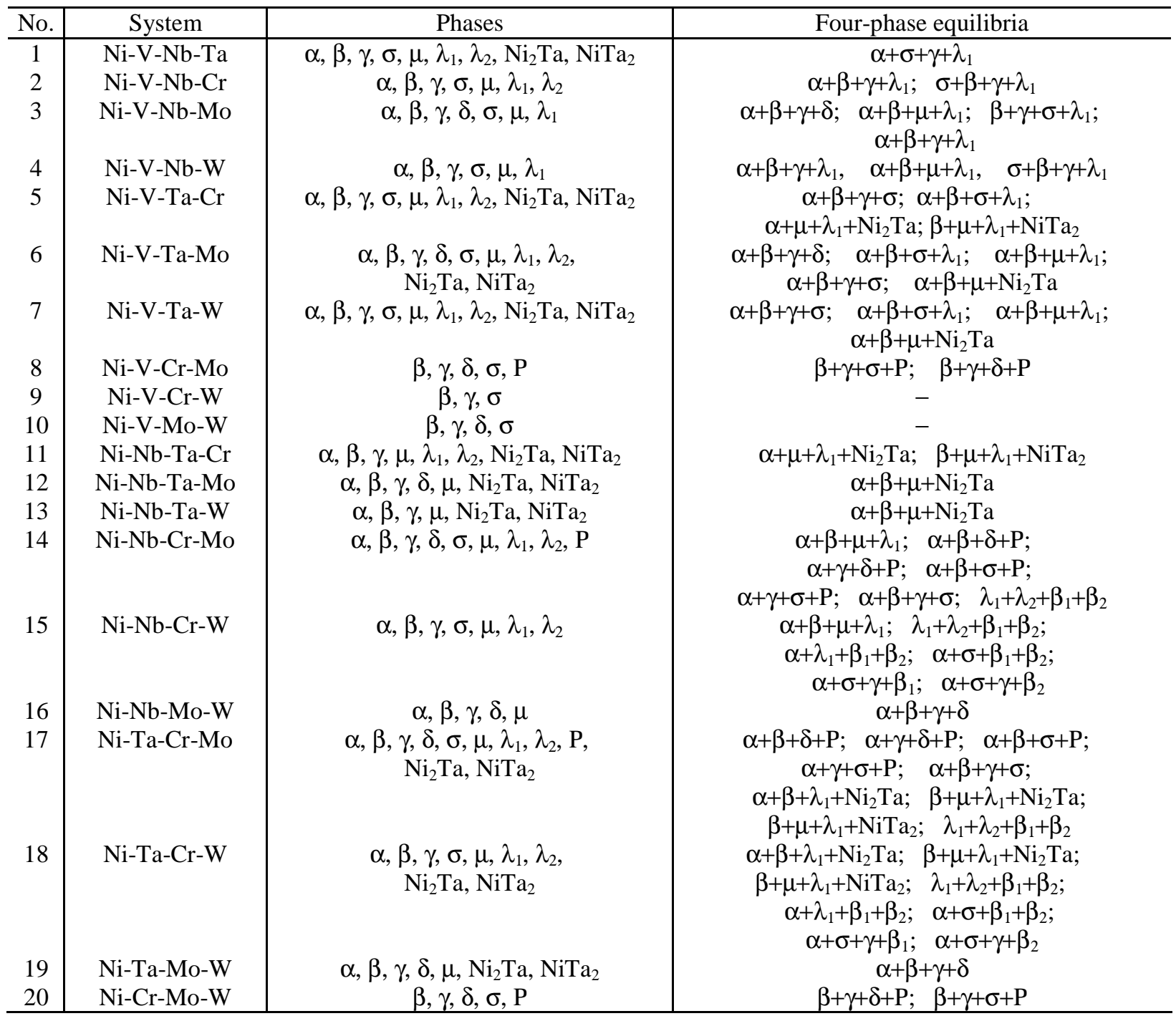

Table 5 Five-phase equilibria in quinary systems on the basis of nickel and refractory metals.

\begin{tabular}{l|c|c}
\hline No. & System & Five-phase equilibria \\
\hline 1 & Ni-V-Nb-Ta-Cr & $\alpha+\beta+\gamma+\sigma+\lambda_{1}$ \\
2 & Ni-V-Nb-Ta-Mo & $\alpha+\beta+\gamma+\sigma+\lambda_{1}$ \\
3 & Ni-V-Nb-Ta-W & $\alpha+\beta+\gamma+\sigma+\lambda_{1}$ \\
4 & Ni-V-Nb-Cr-Mo & $\alpha+\beta+\gamma+\delta+\mathrm{P} ; \alpha+\beta+\gamma+\sigma+\mathrm{P}$ \\
5 & Ni-V-Nb-Cr-W & - \\
6 & Ni-V-Nb-Mo-W & - \\
7 & Ni-V-Ta-Cr-Mo & $\alpha+\beta+\gamma+\delta+\mathrm{P} ; \alpha+\gamma+\sigma+\mathrm{P} ; \alpha+\beta+\mu+\lambda_{1}+\mathrm{Ni}_{2} \mathrm{Ta}^{\mathrm{T}}$ \\
8 & Ni-V-Ta-Cr-W & $\alpha+\mu+\lambda_{1}+\mathrm{Ni}_{2} \mathrm{Ta}$ \\
9 & Ni-V-Ta-Mo-W & - \\
10 & Ni-V-Cr-Mo-W & - \\
11 & Ni-Nb-Ta-Cr-Mo & $\alpha+\beta+\mu+\lambda_{1}+\mathrm{Ni}_{2} \mathrm{Ta}$ \\
12 & Ni-Nb-Ta-Cr-W & - \\
13 & Ni-Nb-Ta-Mo-W & $\alpha+\beta+\gamma+\delta+\mathrm{P} ; \alpha+\beta+\gamma+\sigma+\mathrm{P}$ \\
14 & Ni-Nb-Cr-Mo-W & $\alpha+\beta+\gamma+\delta+\mathrm{P} ; \alpha+\beta+\gamma+\sigma+\mathrm{P}$ \\
\hline
\end{tabular}


remaining graphs was carried out by investigations of the phase composition in 48 alloys and diffusion zones in a few diffusion pairs consisting of multi-phase alloys. The structure and chemical composition of the phases in the equilibrium alloys and diffusion zones were investigated by scanning electron microscopy, electron microprobe analysis, and X-ray diffraction.

The established four-phase equilibria in the quaternary systems based on nickel and refractory metals of groups V and VI are given in Table 4. The five-phase equilibria in the quinary systems that form in the seven-component system Ni-V-Nb-Ta-Cr-Mo$\mathrm{W}$ can be obtained from the analysis of blocks of experimentally established four-phase equilibria in the quaternary systems by the graph method.

The only five-phase equilibria present in the quinary systems are given in Table 5 . The number of systems in which five-phase equilibria are realized is equal to ten. However, the number of types of fivephase equilibrium is equal to four. For the formation of a six-phase equilibrium it is necessary to have six different types of five-phase equilibrium. Consequently, in the Ni-V-Nb-Ta-Cr-Mo-W system at $1375 \mathrm{~K}$ the formation of six-phase equilibria is not possible. Accordingly, a seven-phase equilibrium does not exist either.

\section{References}

[1] E.M. Slyusarenko, V.A. Borisov, M.V. Sofin, E.Yu. Kerimov, A.E. Chastukhin, J. Alloys Compd. 284 (1999) 171.

[2] E.M. Slyusarenko, M.V. Sofin, E.Yu. Kerimov, Mendeleev Commun. 2 (1999)56.

[3] M.V. Sofin, E.Yu. Kerimov, N.A. Bazhanova, Yu.V. Balykova, A.E. Chastukhin, E.M. Slyusarenko, J. Alloys Compd. 321 (2001) 102.

Proceeding of the X International Conference on Crystal Chemistry of Intermetallic Compounds, Lviv, September 17-20, 2007. 